Supporting Information

\title{
Large-area synthesis and patterning of all-inorganic lead halide perovskite thin films and heterostructures
}

Yiliu Wang ${ }^{\dagger}$, Chuancheng Jia ${ }^{\dagger}$, Zheng Fan $^{\S}$, Zhaoyang Lin ${ }^{\dagger}$, Sung-Joon Lee ${ }^{\S}$, Timothy L.

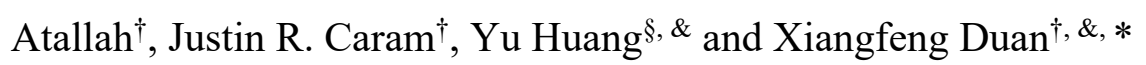

${ }^{\dagger}$ Department of Chemistry and Biochemistry, University of California, Los Angeles, California 90095, United States

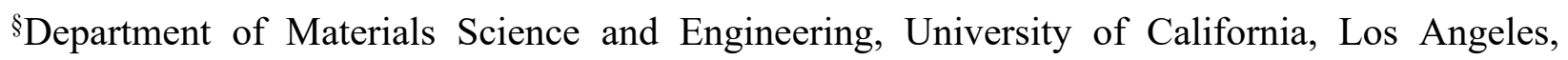
California 90095, United States

${ }^{\&}$ California NanoSystems Institute, University of California, Los Angeles, California 90095, United States

*Correspondence: xduan@chem.ucla.edu (X.D). 


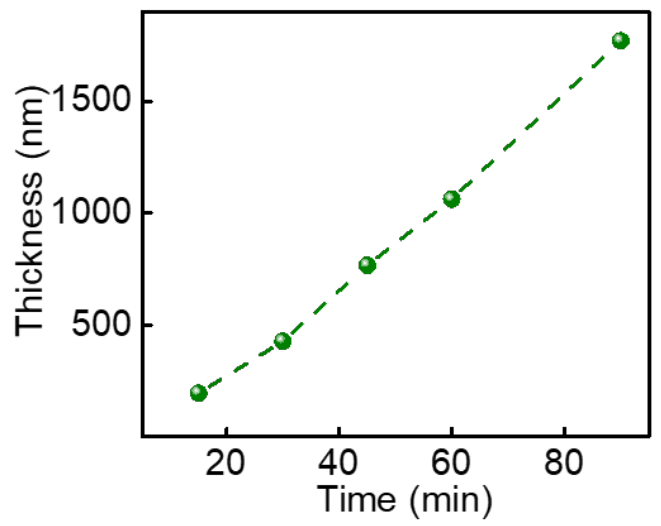

Figure S1. The film thickness of $\mathrm{CsPbBr}_{3}$ vs. growth time. The vertical growth rate was derived to be about $15 \mathrm{~nm} / \mathrm{min}$. 


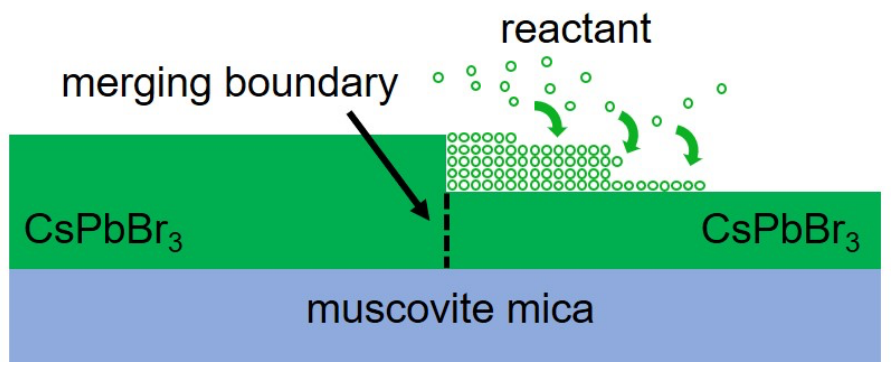

Figure S2. Schematic illustrating the flattening process at the merged domain boundary. 


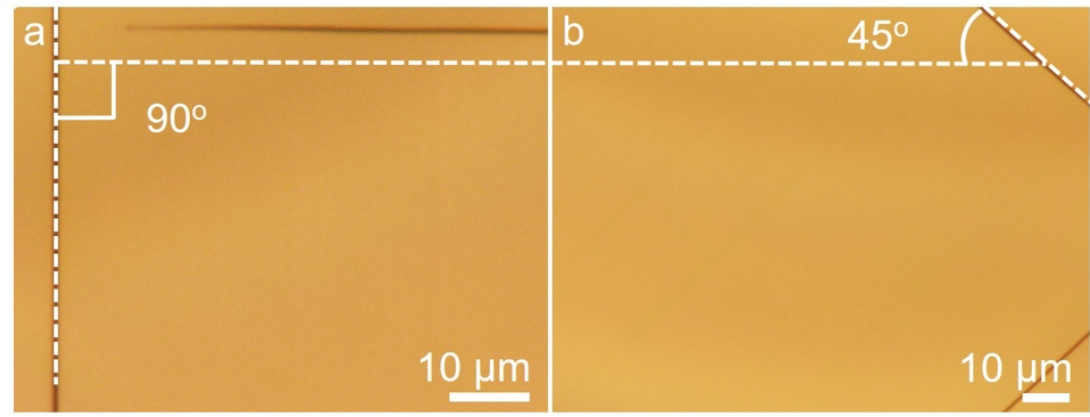

Figure S3. Substrate guided growth of $\mathrm{CsPBr}_{3}$ on two muscovite mica substrates with different orientations from flow direction. The two muscovite mica substrates were cut from the same one to ensure they have the identical crystal orientation, and placed at the same location in the tube furnace for the following growth, with one substrate rotated $45^{\circ}$ in respect to the other. (a) The growth on one muscovite mica, with the gutters indicating the orientation of $\mathrm{CsPbBr}_{3}$; (b) The growth on the other muscovite mica with $45^{\circ}$ rotation from the substrate shown in (a). The gutters in (b) also show a $45^{\circ}$ rotation from that in (a), indicating the orientation of $\mathrm{CsPbBr}_{3}$ is dominated by the orientation of muscovite mica, rather than the gas flow direction. 


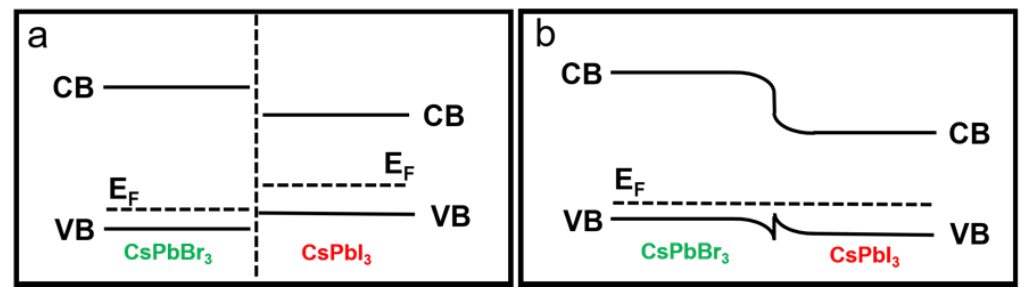

Figure S4. Energy band alignment of $\mathrm{CsPbBr}_{3}-\mathrm{CsPbI}_{3}$ heterostructures. (a) the energy band alignment befroe the equilibrim and (b) the energy band alignment after the equilibrim. 